\title{
Metacarpophalangeal Joint 4
}

National Cancer Institute

\section{Source}

National Cancer Institute. Metacarpophalangeal Joint 4. NCI Thesaurus. Code C102319.

A condyloid synovial joint within the fourth digit of the hand connecting the metacarpal to the proximal phalanx. 